\title{
Data-Driven Distributed Combined Primary and Secondary Control in Microgrids
}

\author{
Seyed Sohail Madani, Christoph Kammer, Alireza Karimi
}

\begin{abstract}
This paper presents a comprehensive data-driven distributed combined primary/secondary controller design method for microgrids. This method provides transient and steady-state performance including power-sharing and voltage and frequency restoration while guaranteeing stability for fixed communication delay. The measured data is directly used for controller design with no need for knowledge about the order or structure of the system and grid physical parameters. Moreover, no assumption is made on $X / R$-ratio of feeders. All the control specifications are formulated as frequency-domain constraints on the 2-norm of weighted sensitivity functions. Then, using a recently developed frequency-domain robust control design method, a distributed fixed-structure controller is synthesized in one step. The performance of the obtained controller is validated using Hardware-In-the-Loop (HIL) experiments. The results show considerable improvement in transient performance, while providing power-sharing and voltage and frequency restoration using distributed implementation.
\end{abstract}

Index Terms-Data-driven controller design, frequency control, voltage control, power-sharing, microgrid control, distributed control.

\section{INTRODUCTION}

G LOBAL warming and imposing limitation on greenhouse gas emission have led to the increase of renewable generation penetration in electrical grids. In order to facilitate the integration of renewable Distributed Generation units (DGs) and Energy Storage Systems (ESSs), the idea of microgrid has been proposed. A microgrid is a small distribution power system that includes its own DGs, ESSs and loads. It has the capability to operate autonomously (islanded mode) or in grid-connected mode. As a consequence of the penetration of renewable energy resources, microgrids suffer from low inertia and high fluctuation of generation. The microgrid control is known as a challenging problem and has been widely studied in the literature [1]. The main expected features of an efficient microgrid control system include providing power-sharing, voltage and frequency steady-state disturbance rejection, voltage and frequency transient performance while guaranteeing stability.

Droop control [2] is the most well-known method for powersharing in microgrids with dominant inductive or resistive

The authors are with the Laboratoire d' Automatique, École Polytechnique Fédérale de Lausanne (EPFL), 1015 Lausanne, Switzerland. (Corresponding author: Alireza Karimi (alireza.karimi@epfl.ch).)

This project is carried out within the frame of the Swiss Centre for Competence in Energy Research on the Future Swiss Electrical Infrastructure (SCCER-FURIES) with the financial support of the Swiss Innovation Agency (Innosuisse - SCCER program). The work of S. S. Madani (e-mail: sohail.madani@epfl.ch) is supported by the Swiss National Science Foundation under Grant 200021_172828. feeders. This method shares power proportionally among DGs based on the frequency deviation and has been popular because of being model-free, very simple and decentralized. However, the major drawbacks are the steady-state errors in voltage and frequency as well as poor transient performance. Moreover, even power-sharing is not achieved for most of distribution grids where the feeders are not dominantly resistive or inductive [1], [3]. Hierarchical control structure [3], [4] is proposed mainly to improve the steady-state voltage and frequency response by adding a secondary control layer, which should be implemented in a centralized [5] or distributed way [6]. However, conventional secondary layer deteriorates the power-sharing and requires time scale separation between different layers. The low bandwidth of higher layers leads to slow voltage and frequency restoration which may trigger the corresponding protection relays. A solution to this problem is proposed in [7] using a distributed averaging control system. The small-signal stability of these systems is studied in [8], [9]. The nonlinear stability is assessed rigorously for lossy systems in [10] and for lossless systems in [7], [11], [12].

Apart from power-sharing and steady-state voltage and frequency performance, designing controllers to improve the transient performance in microgrids is a major challenge. Having mixed resistive/inductive lines, lack of inertia, and poor transient modeling are main factors affecting the transient performance of the microgrids. Different solutions have been proposed in the literature in this regard. In order to improve the transient performance, one idea is to mimic Synchronous Generator (SG) behavior and provide virtual inertia to the system [13]-[15]. While providing inertia, these methods do not use the capability of inverters as high bandwidth actuators. Another idea is to add an $H_{\infty}$ robust controller to the primary droop controller [16]. However, when combined with droop controllers, both approaches are based on the questionable assumption on the dominantly resistive or inductive feeders. The use of quadratic droop control, proposed in [17], can improve the performance of the control system for the distribution grids with uniform $X / R$-ratio of the feeders. Some advanced model-based control techniques combine the primary and secondary control without considering the power-sharing performance [18]-[21].

In the literature, there is a lack of a comprehensive systematic microgrid control synthesis method to provide powersharing in addition to voltage and frequency restoration and at the same time, improving transient performance for arbitrary $X / R$-ratio. For filling the mentioned gap, this paper proposes a non-droop based primary/secondary control design method formulated as a convex optimization problem in a data-driven 
framework. Only measurement data of the power grid is used in controller synthesis and there is no need for parametric system identification, and no knowledge about the physical parameters of the power system is required. The closed-loop stability is guaranteed for the fixed communication delay using specific fixed terms in the controller structure of distributed controllers.

The performance of the proposed method has been validated through HIL experiment on an islanded microgrid including SG, batteries and PV units interfaced with switching inverters. The distributed controllers are implemented on embedded realtime systems and the microgrid is simulated in a real-time simulator.

The rest of the paper is organized as follows: In Section II, the controller design method is reviewed. Section III shows how the performance specifications for the control of a microgrid can be transformed to frequency-domain convex constraints and integrated into the proposed method. The case study and HIL setup results are given in Section IV followed by some concluding remarks.

\section{Control Design By Convex Optimization}

A recently developed control design method which is based on the frequency response of multivariable systems and convex optimization is used in this paper. The method can be employed to design fixed-structure controllers for infinitedimensional systems, which allows the use of frequency response data for controller design. A full theoretical exposition of the method can be found in [22], which is summarized in the sequel.

1) Frequency Response Data: The system to be controlled is a Linear Time-Invariant multivariable (LTI-MIMO) system represented by its frequency response $G(j \omega) \in \mathbb{C}^{n \times m}$, with $m$ inputs and $n$ outputs. $G(j \omega)$ is assumed to be bounded in all frequencies except for a finite set of frequencies $B_{g}$, which correspond to the poles of $G$ on the imaginary axis. Further, define $\omega \in \Omega$ with:

$$
\Omega=\left\{\omega \mid-\frac{\pi}{T_{s}} \leq \omega \leq \frac{\pi}{T_{s}}\right\} \backslash B_{g}
$$

where $T_{s}$ is the sampling time of the control system. In this paper, frequency $\omega$ represents the points in the frequency domain on which Fourier transform is calculated, while the frequency shown by $f$ is the derivative of the electrical angle. For the sake of simplicity, in the rest of the paper, arguments of transfer functions are omitted whenever clear from the context.

2) Controller Structure: Since the design is based on frequency-domain data, it is possible to directly design a discrete-time controller using the frequency response of a continuous-time plant. The controller is defined as $K(z)=$ $X(z) Y(z)^{-1}$, where:

$$
\begin{aligned}
& X(z)=\left(\underline{X}_{\delta} z^{\delta}+\underline{X}_{\delta-1} z^{\delta-1}+\cdots+\underline{X}_{1} z+\underline{X}_{0}\right) \circ F_{X}(z) \\
& Y(z)=\left(I z^{\delta}+\underline{Y}_{\delta-1} z^{\delta-1}+\cdots+\underline{Y}_{1} z+\underline{Y}_{0}\right) \circ F_{Y}(z)
\end{aligned}
$$

$X(z)$ and $F_{X}(z)$ are $m \times n$ and $Y(z)$ and $F_{Y}(z)$ are $n \times n$ polynomial matrices in $z$ and $\circ$ denotes the element-wise matrix multiplication. $\underline{X}_{i} \in \mathbb{R}^{m \times n}$ for $i \in\{1,2, \cdots, \delta\}$ and
$\underline{Y}_{r} \in \mathbb{R}^{n \times n}$ for $r \in\{1,2, \cdots, \delta-1\}$ contain controller parameters. $F_{X}(z)$ and $F_{Y}(z)$ are the fixed terms of controller.

3) Control Performance: It can be defined as the minimization of the weighted norm of any closed-loop sensitivity function. For example, consider the following performance objective on the output sensitivity function $\mathcal{S}=(I+G K)^{-1}$ :

$$
\min _{K}\left\|W_{L} \mathcal{S} W_{R}\right\|_{2}
$$

where $W_{L}$ and $W_{R}$ are the left and right weighting filters and $W_{R}$ is invertible. This objective function, for a stable closed-loop system, can be approximated by a semi-definite programming using a frequency grid $\Omega_{N}=\left\{\omega_{1}, \ldots, \omega_{N}\right\}$, where $\omega_{1} \geq 0$ and $\omega_{N}=\pi / T_{s}$ :

$$
\begin{gathered}
\min \sum_{k=1}^{N} \operatorname{tr}\left(\Gamma_{k}\right) \\
\left(W_{L k} \mathcal{S}_{k} W_{R k}\right)^{*}\left(W_{L k} \mathcal{S}_{k} W_{R k}\right) \leq \Gamma_{k}, \forall\left\{k \mid \omega_{k} \in \Omega_{N}\right\}
\end{gathered}
$$

where a frequency function with subscript $k$ shows the value of the function at $\omega_{k}$ (e.g. $\mathcal{S}_{k}=\mathcal{S}\left(e^{j \omega_{k}}\right)$ ). The optimization variables are the controller parameters (the parameters of $X(z)$ and $Y(z)$ i.e. $\underline{X}_{i}$ for $i \in\{1,2, \cdots, \delta\}$ and $\underline{Y}_{r}$ for $r \in\{1,2, \cdots, \delta-1\}$ ) and dummy matrix variables $\left(\Gamma_{k}>0 \in \mathbb{C}^{n \times n}\right.$ for $\left.k=1, \ldots, N\right)$. Finally, $(\cdot)^{*}$ denotes the conjugate transpose. Replacing $\mathcal{S}$ with $\left(I+G X Y^{-1}\right)^{-1}$, the constraint is reformulated as:

$$
W_{L k} Y_{k}\left(\left(W_{R}{ }_{k}^{-1} M_{k}\right)^{*}\left(W_{R_{k}}^{-1} M_{k}\right)\right)^{-1}\left(W_{L k} Y_{k}\right)^{*} \leq \Gamma_{k}
$$

where $M_{k}=Y_{k}+G_{k} X_{k}$. Taking the Schur complement yields:

$$
\left[\begin{array}{cc}
\Gamma_{k} & W_{L k} Y_{k} \\
\left(W_{L k} Y_{k}\right)^{*} & \left(W_{R_{k}}^{-1} M_{k}\right)^{*}\left(W_{R k}{ }^{-1} M_{k}\right)
\end{array}\right] \geq 0
$$

for $k=1, \ldots, N$. In [22], it is shown that the quadratic part in the lower right can be linearized around $M_{c k}=Y_{c k}+G_{k} X_{c k}$ where $K_{c}=X_{c} Y_{c}^{-1}$ is a stabilizing initial controller. This leads to a convex optimization problem with Linear Matrix Inequality (LMI) constraint as follows:

$$
\begin{gathered}
\min \sum_{k=1}^{N} \operatorname{tr}\left(\Gamma_{k}\right) \\
{\left[\begin{array}{cc}
\Gamma_{k} & W_{L k} Y_{k} \\
\left(W_{L k} Y_{k}\right)^{*} & \mathbf{M}_{W k}
\end{array}\right] \geq 0, \forall\left\{k \mid \omega_{k} \in \Omega_{k}\right\}}
\end{gathered}
$$

where

$$
\begin{aligned}
& \mathbf{M}_{W_{k}}=M_{W k}{ }^{*} M_{W_{c k}}+M_{W_{c k}}{ }^{*} M_{W_{c k}}-M_{W_{c k}}{ }^{*} M_{W_{c k}} \\
& M_{W k}=W_{R_{k}}{ }^{-1} M_{k} \quad ; \quad M_{W_{c k}}=W_{R_{k}}{ }^{-1} M_{c k}
\end{aligned}
$$

If the following conditions are satisfied, the final controller $K$ will be a stabilizing controller:

1) $\operatorname{det}(Y) \neq 0, \forall \omega \in \Omega$.

2) The initial controller $K_{c}$ and the final controller $K$ share the same poles on the stability boundary.

3) The order of $\operatorname{det}(Y)$ is equal to the order of $\operatorname{det}\left(Y_{c}\right)$. 


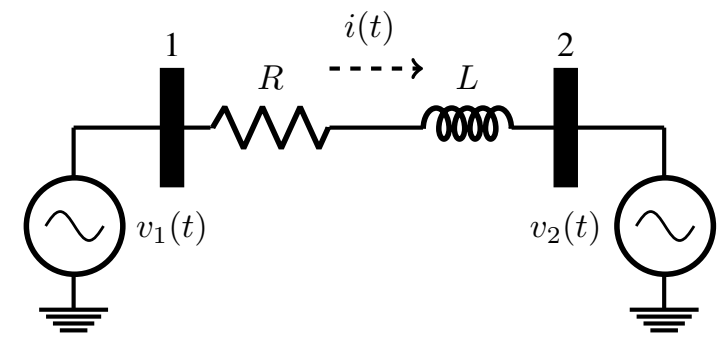

Fig. 1. Single line diagram of a two-node power system

\section{Primary-Secondary Control Design Problem}

In this paper, the frequency response of the Dynamic Phasor Model (DPM) of the microgrid is used for the controller design. For the first time, DPM was proposed in [23] and is used in different areas in power systems and power electronics [24]-[27]. The important difference of DPM with conventional phasor analysis is in considering the time derivative of the phasors. Therefore, DPM can represent the fast electromagnetic dynamics of a network more precisely. In the following section, DPM for a simple two-node power system is presented.

\section{A. Dynamic Phasor Model of a Two-Node System}

Consider a simple two-node system shown in Fig. 1. Active and reactive powers injected from the first bus of this system can be defined as [26]:

$$
\begin{aligned}
& P=\frac{L s+R}{\left|Z_{e}\right|^{2}}\left(V_{1}^{2}-V_{1} V_{2} \cos \Phi_{V_{1}}\right)+\frac{X_{L}}{\left|Z_{e}\right|^{2}}\left(V_{1} V_{2} \sin \Phi_{V_{1}}\right) \\
& Q=\frac{X_{L}}{\left|Z_{e}\right|^{2}}\left(V_{1}^{2}-V_{1} V_{2} \cos \Phi_{V_{1}}\right)-\frac{L s+R}{\left|Z_{e}\right|^{2}}\left(V_{1} V_{2} \sin \Phi_{V_{1}}\right)
\end{aligned}
$$

where $X_{L}:=L\left(2 \pi f_{n}\right),\left|Z_{e}\right|:=\sqrt{(L s+R)^{2}+X_{L}^{2}}$. It is assumed that bus number 2 is considered as the reference for angle, which means $\Phi_{V_{2}}=0$. By linearizing (7) around the equilibrium voltage $V_{1_{e}}$, the transfer function between active and reactive power deviations and local frequency and voltage deviations, can be written as:

$$
\left[\begin{array}{l}
P \\
Q
\end{array}\right]=\left[\begin{array}{cc}
\frac{X_{L} V_{1 e}^{2}}{s\left|Z_{e}\right|^{2}} & \frac{(L s+R) V_{1 e}}{\left|Z_{e}\right|^{2}} \\
-\frac{(L s+R) V_{1 e}^{2}}{s\left|Z_{e}\right|^{2}} & \frac{X_{L} V_{1 e}}{\left|Z_{e}\right|^{2}}
\end{array}\right]\left[\begin{array}{c}
f \\
V
\end{array}\right]
$$

In conventional power system analysis, the impact of $L s$ is usually ignored, which leads to inaccurate electromagnetic transient dynamics. Furthermore, in distribution grids, the value of $R$ is not negligible, thus the system is coupled.

\section{B. Input/Output Definition for Microgrid}

As a general case, assume a power system including $\kappa$ DGs and $\ell$ loads. The vector of desired active power of all DGs can be defined as $\bar{P}_{D G}:=\left[\bar{p}_{1}, \ldots, \bar{p}_{\kappa}\right]^{\top}$. The vector of measured active power of DGs (i.e. $P_{D G}$ ) is defined similarly. The active power error vector can be defined as $P_{e}=\bar{P}_{D G}-P_{D G}$. With similar convention, vector of desired reactive power (i.e.
TABLE I

DESCRIPTION OF THE TRANSFER FUNCTIONS USED IN FIG. 2

\begin{tabular}{l|l}
\hline$K_{P I D}$ & SG internal speed controller \\
\hline$G_{S, m}$ & $\begin{array}{l}\text { from mechanical reference of } \\
\text { SG to its mechanical angular frequency }\end{array}$ \\
\hline$G_{S, e}$ & $\begin{array}{l}\text { from SG output } \\
\text { electrical power to its mechanical angular frequency }\end{array}$ \\
\hline$G_{I, f}$ & from inverter frequency reference to its frequency \\
\hline$G_{I, V}$ & from inverter voltage reference to its output voltage \\
\hline$G_{S, V}$ & equivalent closed-loop response of SG AVR \\
\hline$G_{g r i d}$ & $\begin{array}{l}\text { from the power grid nodal voltage and } \\
\text { frequency to injected active active and reactive power }\end{array}$ \\
\hline$G_{d}$ & from load power to power drawn at DG nodes \\
\hline$K$ & MIMO controller \\
\hline
\end{tabular}

$\bar{Q}_{D G}$ ), vector of measured reactive power (i.e. $Q_{D G}$ ), vector of reactive power error (i.e. $Q_{e}$ ), vector of desired frequency (i.e. $\bar{f}_{D G}$ ) and vector of desired voltage (i.e. $\bar{V}_{D G}$ ) of DGs can be defined. The vector of active power of loads is defined as $P_{L}:=\left[p_{L_{1}}, \ldots, p_{L_{\ell}}\right]^{\top}$ and similarly the vector of load reactive power (i.e. $\bar{Q}_{L}$ ) can be defined. Accordingly, the input/output relation of the MIMO plant corresponding to the power system ( $G_{\text {comp }}$ in Fig. 2) can be written as:

$$
\left[P_{D G}^{\top}, Q_{D G}^{\top}\right]^{\top}-\left[P_{d}^{\top}, Q_{d}^{\top}\right]^{\top}=G_{\text {comp }}\left[\bar{f}_{D G}^{\top}, \bar{V}_{D G}^{\top}\right]^{\top}
$$

where $\left[P_{d}^{\top}, Q_{d}^{\top}\right]^{\top}$ is output power disturbance vector. Using DPM, the complete model of the grid including line power flow and the elements in the grid such as VSI and the SG is proposed in [28]. The block diagram of a general microgrid is depicted in Fig. 2 and its parameters are explained in Table I.

\section{Proposed Controller Design Method}

The design method includes the following steps:

1) Performance in Disturbance Rejection: To reduce the impact of disturbances, the weighted 2-norm of the output sensitivity function $\mathcal{S}=\left(I+G_{\text {comp }} K\right)^{-1}$ can be minimized:

$$
\min _{K}\left\|W_{1} \mathcal{S}\right\|_{2}
$$

where $W_{1}$ is the output sensitivity weighting filter. Since the disturbances in power systems are mostly in the form of connection or disconnection of the loads, the step response of sensitivity function has high importance. Consequently, considering the relation of 2-norm in time-domain and frequencydomain, the 2-norm of low-pass filtered sensitivity function is minimized in order to reduce the time-domain oscillation generated by low-frequency disturbances.

2) Active Power-Sharing: Proportional active powersharing can be achieved by investigating the sub-matrix of sensitivity function which relates the active power error to active power disturbance. This disturbance is the impact of the changes in active power loads on the drawn power at the DG bus. The sensitivity function $\mathcal{S}$ is split into 4 parts:

$$
\left[\begin{array}{l}
P_{e} \\
Q_{e}
\end{array}\right]=\left[\begin{array}{ll}
S_{11} & S_{12} \\
S_{21} & S_{22}
\end{array}\right]\left[\begin{array}{c}
P_{d} \\
Q_{d}
\end{array}\right]
$$

$S_{11}$ defined above is the transfer function of active power disturbance to active power tracking error. For example, assume an active power disturbance $p_{d_{i}}$ is applied to DG node $i$ :

$$
P_{e}=S_{11}\left[\ldots, p_{d_{i}}, \ldots\right]^{\top}
$$




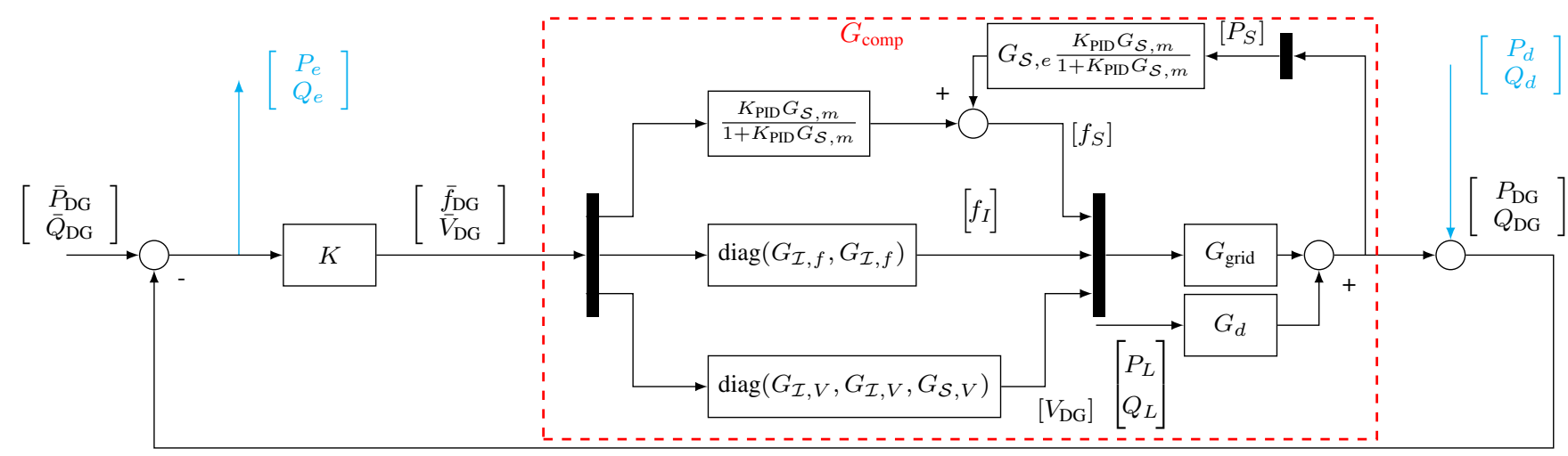

Fig. 2. Block diagram of complete model of the power system

Then, if power is shared proportionally, the following steadystate tracking errors should be obtained:

$$
p_{e_{j}}=\frac{p_{n_{j}}}{p_{\text {tot }}} p_{d_{i}} \text { for } j=1, \ldots, \kappa
$$

where $p_{n_{j}}$ is the nominal power of the $j$-th DG unit and $p_{\text {tot }}=$ $p_{n_{1}}+\cdots+p_{n_{\kappa}}$. The same relation should also hold for the other disturbances, which means $\left.S_{11}(j \omega)\right|_{\omega=0}$ should take the following value:

$$
S_{11}^{0}=\frac{1}{p_{\text {tot }}}\left[\begin{array}{ccc}
p_{n_{1}} & \cdots & p_{n_{1}} \\
\vdots & \ddots & \vdots \\
p_{n_{\kappa}} & \cdots & p_{n_{\kappa}}
\end{array}\right]
$$

This leads to the following constraint:

$$
\left.S_{11}(j \omega)\right|_{\omega=0}=S_{11}^{0}
$$

According to (11) and (15), $S_{11}$ is needed for power-sharing. To formulate the constraints in form of (3), the following transformation can be applied to $\mathcal{S}$ :

$$
\left[\begin{array}{ll}
S_{11} & \sigma S_{12}
\end{array}\right]=\underbrace{\left[\begin{array}{ll}
I & 0
\end{array}\right]}_{W_{L}} \mathcal{S} \underbrace{\operatorname{diag}(I, \sigma I)}_{W_{R}}
$$

where $\sigma \in \mathbb{R}$ is a small number to make $W_{R}$ invertible. Using this transformation

$$
\left\|W_{L} \mathcal{S} W_{R}\right\|_{2}^{2}=\left\|S_{11}\right\|_{2}^{2}+\sigma^{2}\left\|S_{12}\right\| \approx\left\|S_{11}\right\|_{2}^{2}
$$

As the power-sharing problem is focused on steady-state, the first frequency is the most important point. Consequently, the problem of power-sharing can be written as:

$$
\min _{K}\left\|W_{L 1} \mathcal{S}_{1} W_{R 1}-\left[\begin{array}{ll}
S_{11}^{0} & 0
\end{array}\right]\right\|_{2}
$$

3) Frequency and Voltage Performance: Usually when the loads or the references for DG power change, voltage and frequency of the system deviate from the nominal values. To minimize the deviation in frequency and voltage as well as the frequency steady-state error, weighted 2-norm of the input sensitivity function $\mathcal{U}=K\left(I+G_{\text {comp }} K\right)^{-1}$ can be minimized.

$$
\min _{K}\left\|W_{2} \mathcal{U}\right\|_{2}
$$

where $W_{2}$ is the input sensitivity weighting filter and the following relation holds for $\mathcal{U}$ :

$$
\left[\bar{f}_{\mathrm{DG}}^{\top}, \bar{V}_{\mathrm{DG}}^{\top}\right]^{\top}=\mathcal{U}(j \omega)\left[P_{d}^{\top}, Q_{d}^{\top}\right]^{\top}
$$

In general, at each frequency point, a higher weight will result in a lower deviation. Particularly, in order to reduce the frequency steady-state error $W_{2}$ should have high gain at low frequencies.

4) Complete Design Problem: Combining the mentioned specifications leads to the following multi-objective optimization problem:

$$
\begin{gathered}
\min _{K} \underbrace{\left\|W_{1} \mathcal{S}\right\|_{2}}_{\text {Disturbance Rejection }}+\underbrace{\left\|W_{2} \mathcal{U}\right\|_{2}}_{\text {Freq./Volt. Performance }} \\
\text { s.t. }\left.S_{11}\left(j \omega_{k}\right)\right|_{k=1}=S_{11}^{0} \quad \text { (Power-Sharing) }
\end{gathered}
$$

Then, using the convex formulation from (6), the robust control design problem in (21) can be written as a convex optimization problem with LMI constraints:

$$
\begin{aligned}
& \min \underbrace{\alpha \operatorname{tr}\left(\Gamma_{P 1}\right)}_{\text {Power-Sharing }}+\sum_{k=1}^{N}[\underbrace{\operatorname{tr}\left(\Gamma_{S_{k}}\right)}_{\text {Dist. Rej. }}+\underbrace{\operatorname{tr}\left(\Gamma_{U k}\right)}_{\text {Freq./Volt. Perf. }}] \\
& {\left[\begin{array}{cc}
\Gamma_{S k} & W_{1 k} Y \\
\left(W_{1 k} Y_{k}\right)^{*} & M_{k}^{*} M_{c k}+M_{c k}^{*} M_{k}-M_{c k}^{*} M_{c k}
\end{array}\right]>0,} \\
& {\left[\begin{array}{cc}
\Gamma_{U k} & W_{2 k} X_{k} \\
\left(W_{2 k} X_{k}\right)^{*} & M_{k}^{*} M_{c k}+M_{c k}^{*} M_{k}-M_{c k}^{*} M_{c k}
\end{array}\right]>0,} \\
& {\left[\begin{array}{cc}
\Gamma_{P 1} & B \\
B^{*} & \mathbf{M}_{W 1}
\end{array}\right]>0} \\
& Y_{k}^{*} Y_{c k}+Y_{c k}^{*} Y_{k}-Y_{c k}^{*} Y_{c k}>0 \text {, }
\end{aligned}
$$

for all $k=1, \ldots, N$, where $B=W_{L_{1}} Y_{1}-\left[\begin{array}{ll}S_{11}^{0} & 0\end{array}\right] W_{R_{1}}^{-1} M_{1}$. The scalar $\alpha$ is a weighting factor that denotes the importance of the power-sharing, and $\Gamma_{S k}>0, \Gamma_{U k}>0$ are auxiliary positive definite matrix variables as defined for the output and input sensitivity functions corresponding to $k^{\text {th }}$ frequency point, respectively. In the same way, $\Gamma_{P 1}>0$ is the auxiliary positive definite matrix variable defined for the power-sharing at steady-state $\left(\omega_{1}\right)$. The necessary and sufficient condition for the first stability constraint (i.e. $\operatorname{det}(Y) \neq 0, \forall \omega \in \Omega$ ) is $Y^{*} Y>0$. Since this constraint is not convex, it can be linearized around the initial controller as $Y_{c}^{*} Y+Y^{*} Y_{c}$ $Y_{c}^{*} Y_{c}>0$. The last constraint in (22) is added to satisfy this constraint for all frequencies. Since the problem is an approximation of the original non-convex problem, the obtained solution depends on the initial controller. Therefore, an 
iterative approach is used, where the optimization problem is solved multiple times using the calculated controller $K$ of the previous step as the new initial controller $K_{c}$. This choice always guarantees closed-loop stability (assuming the initial choice of $K_{c}$ is stabilizing). Since the objective function is non-negative and non-increasing, the iteration converges to a locally optimal solution of the original non-convex problem.

It can be shown that for each stable plant one choice for stabilizing initial controller is $K_{c}=\epsilon I$ provided that $\epsilon$ is sufficiently small. In order to satisfy the third condition of stability, the initial controller matrices can be selected as:

$$
X_{c}=\epsilon z^{\delta}, Y_{c}=I z^{\delta}
$$

It should be mentioned that for selecting the order (i.e. $\delta$ ) in designing the controller, the order of the controller is set to a very low value (say 2 or 3 ) initially. If the performance using this order is not satisfactory, the order is increased by one and the design procedure is reiterated.

5) Communication Graph: The controller design method proposed in this paper is capable of handling different structure of communication systems by choosing centralized, distributed or decentralized controller structure. If there is no data communication between two nodes, the corresponding elements will be substituted by zero in $X$ matrix. As an illustrative example, consider a microgrid with three $\mathrm{DGs}\left(\mathrm{DG}_{1}, \mathrm{DG}_{2}\right.$, $\mathrm{DG}_{3}$ ). Controller of each DG calculates its command signals based on the local measurements and measurements transmitted from other DGs. Assume that the available infrastructure provides communication links $\mathrm{DG}_{1}-\mathrm{DG}_{2}$ and $\mathrm{DG}_{2}-\mathrm{DG}_{3}$ but no communication link between $\mathrm{DG}_{1}$ and $\mathrm{DG}_{3}$. Therefore the following structure should be considered for $\underline{X}_{i}$ for $i \in\{1,2, \cdots, \delta\}$ :

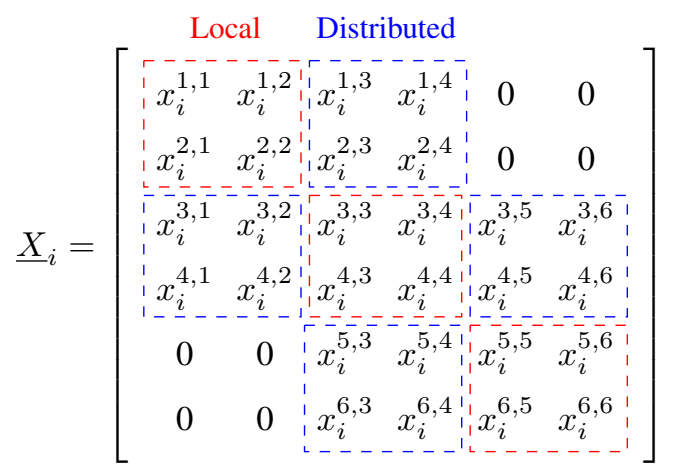

6) Communication Delay: The proposed method is capable of considering communication delay in controller design by defining it as a fixed term in the controller. Assume that there is $d$-sampling time delay for data transmission from node $i$ to node $j$. Then, by multiplying both $(i, j)$-th and $(j, i)$-th element of $F_{X}$ by $z^{-d}$, the impact of communication delay can be considered in the controller design. In order to clarify the idea, in the above-mentioned illustrative example, assume that each communication link has $d$ samples delay. This delay can be included in the fixed term of the controller as follows:

$$
F_{X}(z)=\left[\begin{array}{ccc}
\mathbf{1}_{2 \times 2} & z^{-d} \mathbf{1}_{2 \times 2} & \mathbf{0}_{2 \times 2} \\
z^{-d} \mathbf{1}_{2 \times 2} & \mathbf{1}_{2 \times 2} & z^{-d} \mathbf{1}_{2 \times 2} \\
\mathbf{0}_{2 \times 2} & z^{-d} \mathbf{1}_{2 \times 2} & \mathbf{1}_{2 \times 2}
\end{array}\right]
$$

TABLE II

LINE PARAMETERS

\begin{tabular}{l|l|l||l|l|l}
\hline Line & $\mathbf{R}[\Omega]$ & $\mathbf{X}[\Omega]$ & Line & $\mathbf{R}[\Omega]$ & $\mathbf{X}[\Omega]$ \\
\hline $1-2$ & 0.018 & 0.0034 & $4-8$ & 0.09 & 0.017 \\
$1-3$ & 0.018 & 0.0034 & $8-9$ & 0.045 & 0.0085 \\
$2-7$ & 0.15 & 0.11 & $5-6$ & 0.09 & 0.017 \\
$2-10$ & 0.3 & 0.22 & $6-7$ & 0.3 & 0.22 \\
$3-4$ & 0.45 & 0.085 & $9-10$ & 0.3 & 0.22 \\
$3-5$ & 0.3 & 0.22 & & & \\
\hline
\end{tabular}

\begin{tabular}{|c|c|}
\hline \multicolumn{2}{|l|}{ BESS } \\
\hline Bus: & {$[8,10]$} \\
\hline Output filter Parameters: & $\begin{array}{l}R_{t}=10 \mathrm{~m} \Omega, L_{t}=450 \mu \mathrm{H} \\
R_{g}=58 \mathrm{~m} \Omega, L_{g}=420 \mu \mathrm{H}, C_{f}=50 \mu \mathrm{F}\end{array}$ \\
\hline Time Constants: & $\tau_{\omega}=5 \cdot 10^{-4}, \tau_{U}=5 \cdot 10^{-4}$ \\
\hline Nom. apparent power: & {$[70,40] \mathrm{KVA}$} \\
\hline \multicolumn{2}{|l|}{ Synchronous Generator } \\
\hline Bus: & [5] \\
\hline Inertia Constant: & $\mathrm{H}=1.5$ \\
\hline Internal Impedance: & $R_{o}=19 \mathrm{~m} \Omega, L_{o}=2.7 \mathrm{mH}$ \\
\hline Time Constants: & $\tau_{m}=0.1, \tau_{U}=0.05$ \\
\hline Speed Controller: & $k_{p}=3.18, k_{i}=4.77, k_{d}=0.8, T_{f}=0.05$ \\
\hline Nom. apparent power: & $70 \mathrm{KVA}$ \\
\hline \multicolumn{2}{|l|}{ Inverter } \\
\hline Bus: & {$[1,2,7,8,10]$} \\
\hline parameters: & Switching Freq.: $6.25 \mathrm{KHz}$, DC Voltage: $500 \mathrm{~V}$ \\
\hline \multicolumn{2}{|l|}{ PV } \\
\hline Bus: & {$[1,2,7]$} \\
\hline Output filter Parameters: & $\begin{array}{l}R_{t}=10 \mathrm{~m} \Omega, L_{t}=450 \mu \mathrm{H} \\
R_{g}=58 \mathrm{~m} \Omega, L_{g}=420 \mu \mathrm{H}, C_{f}=50 \mu \mathrm{F}\end{array}$ \\
\hline Active Power: & $10 \mathrm{~kW}$ \\
\hline \multicolumn{2}{|l|}{ Loads } \\
\hline Bus: & {$[3,4,6,9]$} \\
\hline Active/Reactive Power: & {$[30,20,25,45] \mathrm{kW} /[0,0,0,0] \mathrm{VAr}$} \\
\hline
\end{tabular}

TABLE III

DG UNITS PARAMETERS

\section{CASE Study}

The proposed method is applied to a microgrid illustrated in Fig. 3, which is a $50 \mathrm{~Hz} / 230 \mathrm{~V}$ islanded grid including two VSI-interfaced Battery Energy Storage Systems (BESSs), one SG, three PV units in current-controlled mode and four constant-power loads. The lines are either resistive with $\mathrm{X} / \mathrm{R}$ ratio of 0.18 or mixed with $\mathrm{X} / \mathrm{R}$-ratio close to 1 . Furthermore, each VSI is filtered with an LCL-type output filter. The SG is operated in speed control mode and is equipped with an internal speed controller. The parameters of lines and DGs are given in Table II and Table III, respectively.

\section{A. Frequency Response Function}

In this paper, the measurement data is provided through numerical real-time simulation of the grid including the switching inverters. To simplify the design, all controller inputs and outputs are assumed to be normalized to per unit with $V_{\text {base }}=325 \mathrm{~V}(=230 \sqrt{2}), f_{\text {base }}=50 \mathrm{~Hz}, S_{\text {base }}=100 \mathrm{kVA}$. In the operating point, DGs are working in 50 percent of their nominal powers. A Pseudo-Random Binary Sequence (PRBS) signal with small magnitude is added to the closed-loop system references (i.e. $\left[\bar{P}_{D G}, \bar{Q}_{D G}\right]^{\top}$ ) while operating in normal condition and the output of the plant (i.e. $\left[P_{D G}, Q_{D G}\right]^{\top}$ ) are measured. Ten periods of an 8-order PRBS with a sampling time of $5 \mathrm{~ms}$ and a magnitude of 0.05 p.u. is applied. The last three periods of the excitation signal added to the reference of the active power of SG and the actual power injected to the grid at bus No. 5 are shown in Fig. 4.

The frequency response of the closed-loop transfer function $\mathcal{T}=G_{\text {comp }} K\left(I+G_{\text {comp }} K\right)^{-1}$ can be computed using Fourier 


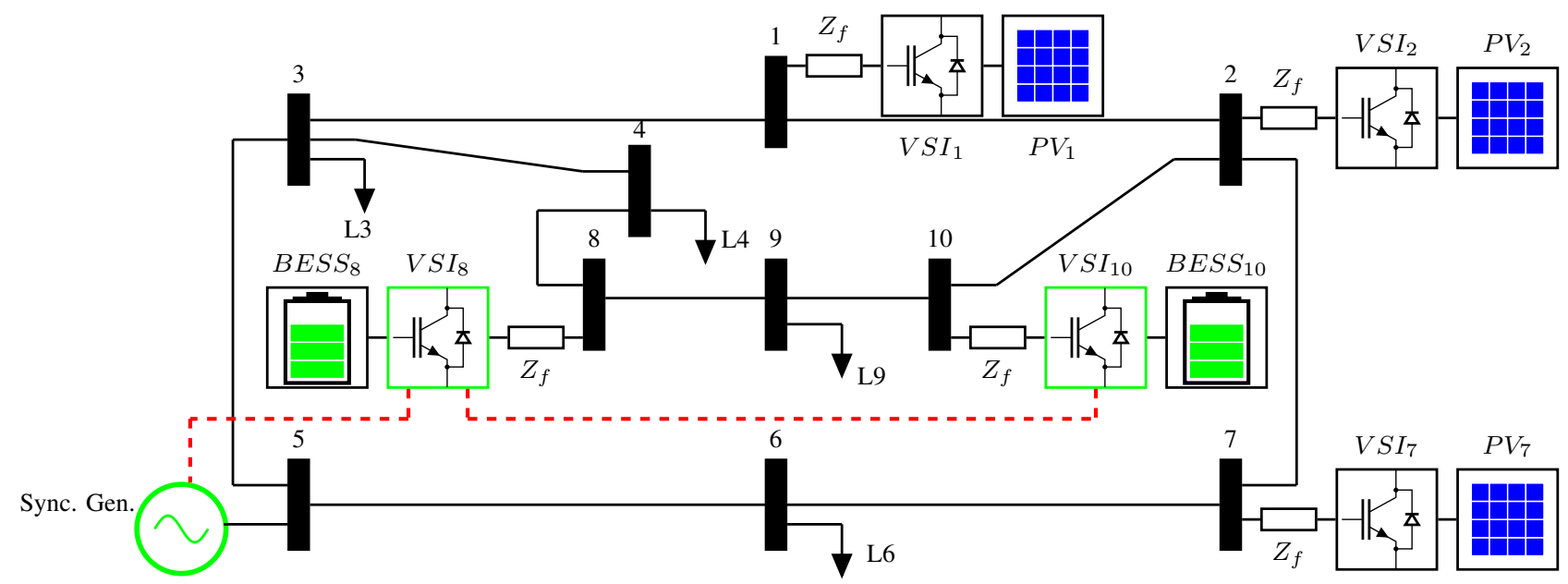

Fig. 3. Single-line diagram of microgrid (solid line: power line, dashed line: communication, $L_{i}$ : load number $i, Z_{f}$ : LCL filter)

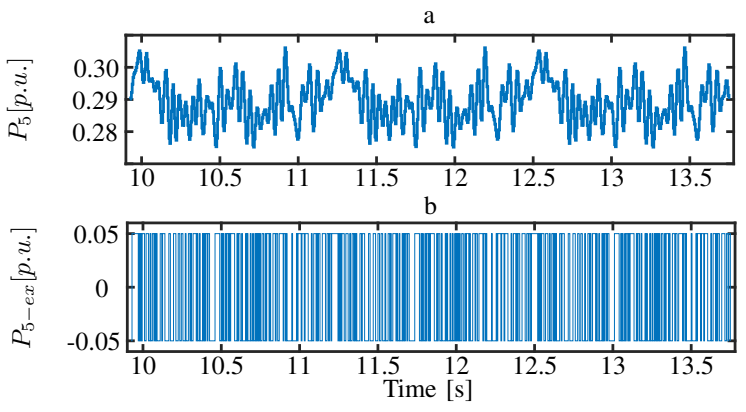

Fig. 4. a) Output power at bus No 5, b) Excitation added to ref. of SG

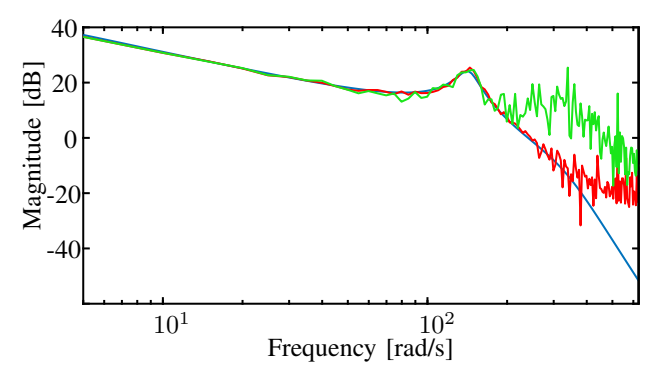

Fig. 5. Frequency response of the active power at bus No. 5 to the frequency reference of the SG at bus No. 5 with linear inverters (red), switching inverters (green) and the linearized parametric model using DPM (blue)

transform of input and output data. Then $G_{\text {comp }}$ can be calculated as

$$
G_{\text {comp }}\left(j \omega_{k}\right)=\mathcal{T}\left(j \omega_{k}\right)\left(K\left(j \omega_{k}\right)\left(I-T\left(j \omega_{k}\right)\right)\right)^{-1}
$$

The results of frequency response calculated using the measurement data are compared with parametric DPM. As an example, the response of active power injected to bus number 5 to the frequency reference of the SG is shown in Fig. 5

\section{B. Controller Design}

An 8-th order controller is designed for the grid in Fig. 3 by solving the convex optimization problem given in (22). For this example, $\epsilon=0.01$ is sufficiently small to achieve the desired performance while avoiding numerical problems. The weighting factor $\alpha$ is chosen to be 1000 . The weighting filters are chosen as $W_{1}^{-1}=1.3 s /(250+s) I$ and $W_{2}^{-1}=\beta I$ where $\beta=0.01$ for $\omega<100 \mathrm{rad} / \mathrm{s}$ and $\beta=0.1$ for $\omega>100 \mathrm{rad} / \mathrm{s}$. Two sampling delays are considered for data transmission between $K_{8}$ and $K_{10}$. The optimization problem is then formulated in Matlab using Yalmip [29] and solved using Mosek [30]. The whole procedure of experiment (using power system measurement of the real-time simulation), frequency response calculation and controller design (using a desktop computer) take less than 3 minutes for this example.

\section{Hardware-In-the-Loop validation}

To validate the performance of the obtained controller, the grid shown in Fig. 3 is simulated in HIL setup including an Opal-rt real-time simulator and MyRIO controllers provided by National Instruments. In contrast with most of validations in the literature, which use simplified average models, the inverters of the batteries are simulated as the switching elements which inject harmonics to the system. The controller for inverters in bus No. $8\left(K_{8}\right)$ and bus No. $10\left(K_{10}\right)$ are separately implemented on two MyRIOs coded by LABVIEW as distributed controllers. Two communication types are employed in this HIL setup:

1) Sharing the data between $K_{8}$ and $K_{10}$ using User Datagram Protocol (UDP) on WiFi with no guarantee for safe transmission of the data, which is the case when the available communication resources are limited in the actual implementation.

2) Sending the local measurement values calculated in Opal-rt real-time simulator to the controller using the analog noisy signals.

Moreover, to have a realistic experimental result, no synchronization signal between opal and two controllers are employed. This results in asynchronous operation of two controllers, which is the case in actual power systems.

The results of the proposed Data-driven Distributed Primary Secondary (DDPS) controller, the classical droop controller 


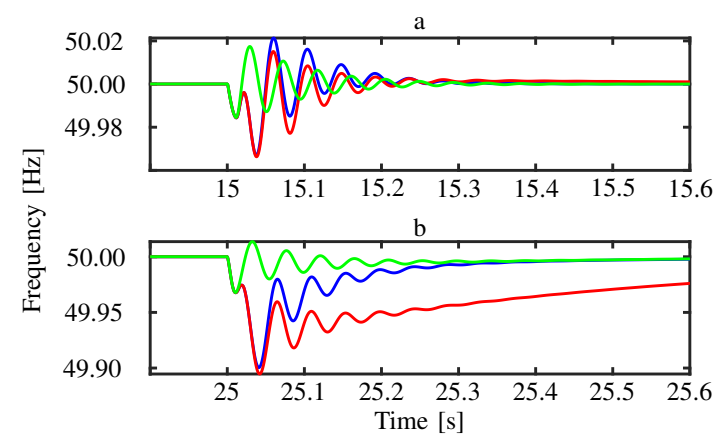

Fig. 6. SG frequency using different methods in a) impulse and b) step disturbance (red: droop with a central integrator, blue: DAPI, green: DDPS)

TABLE IV

FREQUENCY CONTROL COMPARISON

\begin{tabular}{l||c|c}
\hline Method & Frequency nadir [mHz] & Settling time*[ms] \\
\hline Droop & 106 & 690 \\
DAPI & 100 & 181 \\
DDPS & 34 & 17 \\
\hline \multicolumn{2}{|c}{ he settling time is defined as the time that the frequency has less than } \\
mHz frequency drop after addition of load and stays in that condition.
\end{tabular}

with centralized secondary control, and droop controller with a Distributed Averaging PI (DAPI) [7] are compared. In this experiment, a load with double size of $L_{4}$ has been connected at $\mathrm{t}=15 \mathrm{~s}$ for $0.01 \mathrm{~s}$ at bus number 4 to show the impact of impulsive loads and $L_{6}$ is stepped up from $25 \mathrm{~kW}$ to $45 \mathrm{~kW}$ at $\mathrm{t}=25 \mathrm{~s}$ to show steady-state disturbance damping. In Fig. 6, the instantaneous frequency at SG bus after the impulsive and step load is shown. It can be seen that with droop and DAPI controllers, the SG frequency experiences significant oscillations and it takes a long time until the nominal frequency is recovered. The results have been summarized in Table IV. The controller designed in this paper is able to reduce the frequency nadir and its settling time considerably.

The frequency at bus No. 8 and 10, where the BESSs are located, achieved by droop controller, DAPI controller, and controller designed by the proposed method have been shown in Fig. 7 and Fig. 8, respectively. It can be observed that the frequency with the proposed controller has a very low sensitivity to disturbances in comparison to other methods. Since there is no decoupling, both frequency and voltage contribute to disturbance rejection which results in less frequency oscillation at VSI nodes. The superiority of considering the couplings in controller design and also taking advantage of the independence of VSI frequency from physical states can be seen obviously by applying the proposed method.

Fig. 9 shows the active output power of the DGs and proportional power-sharing.

The voltage magnitude at a PV, Battery and SG buses using different methods are compared in Fig. 10 and their normalized two-norm are compared in Table $\mathrm{V}$, which shows improvement

TABLE V

2-NORM OF VOLTAGE DEVIATION AT DIFFERENT BUSES

\begin{tabular}{l||c|c|c}
\hline & bus \#2 & bus \#5 & bus \#8 \\
\hline Droop & 6.60 & 6.62 & 4.69 \\
DAPI & 6.64 & 6.61 & 4.69 \\
DDPS & 5.60 & 5.84 & 3.92 \\
\hline
\end{tabular}

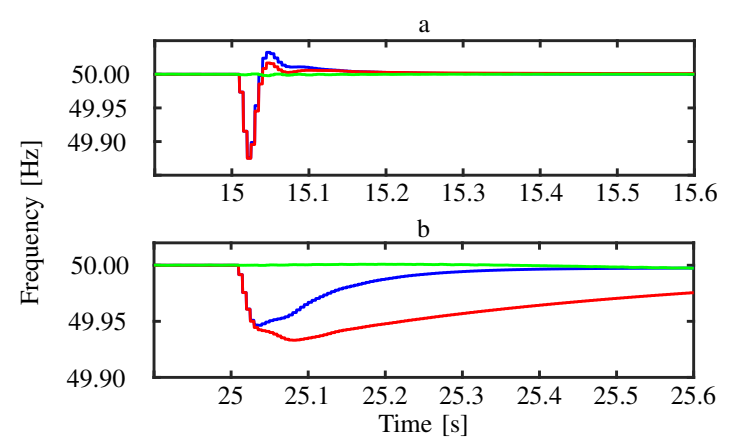

Fig. 7. VSI 8 frequency applying different methods in a) impulse and b) step disturbance (red: droop with central integrator, blue: DAPI, green: DDPS)

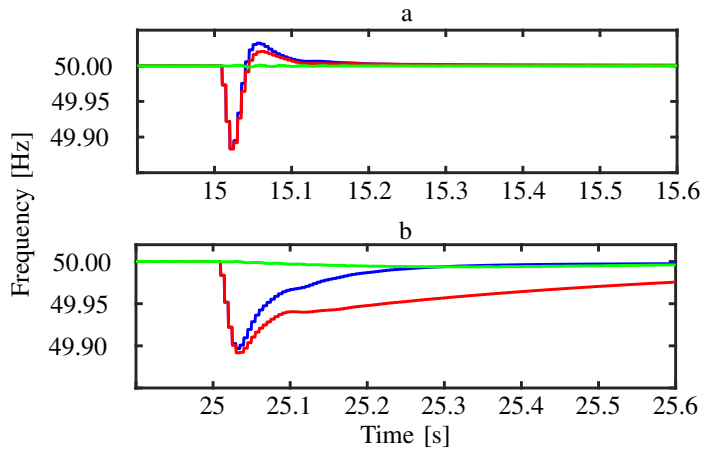

Fig. 8. VSI 10 frequency applying different methods in a) impulse and b) step disturbance (red: droop with central integrator, blue: DAPI, green: DDPS)

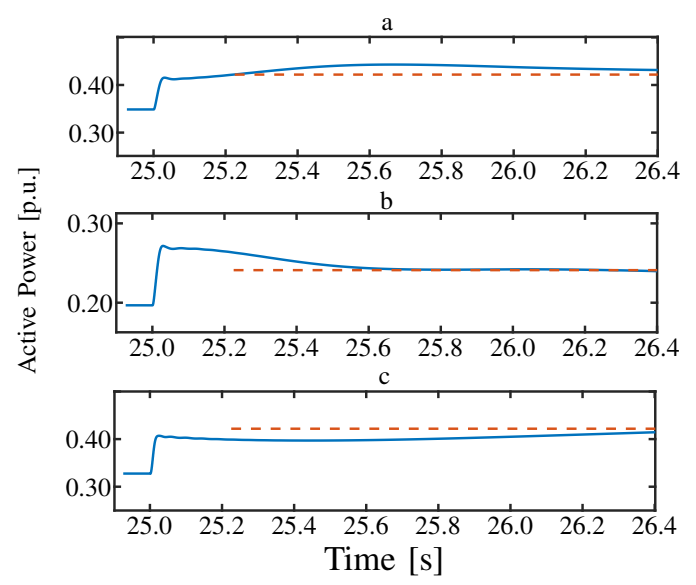

Fig. 9. Active power-sharing using DDPS a) $V S I_{8}$, b) $V S I_{10}$, c) SG (solid line: active power, dashed line: the proportionally shared active power)

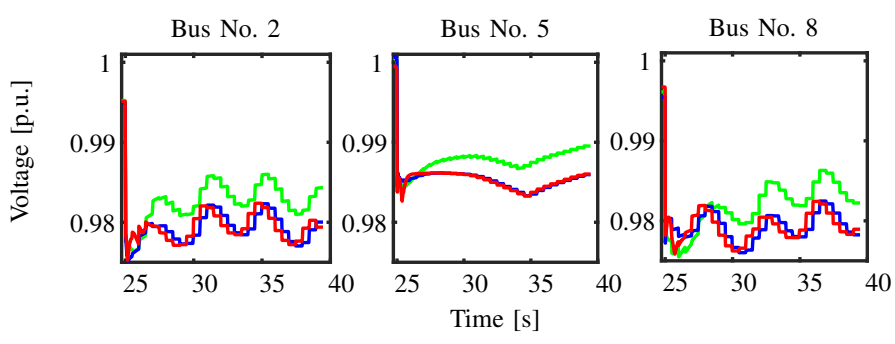

Fig. 10. Bus voltages applying different methods in a) impulse and b) step disturbance (red: droop with central integrator, blue: DAPI, green: DDPS) 


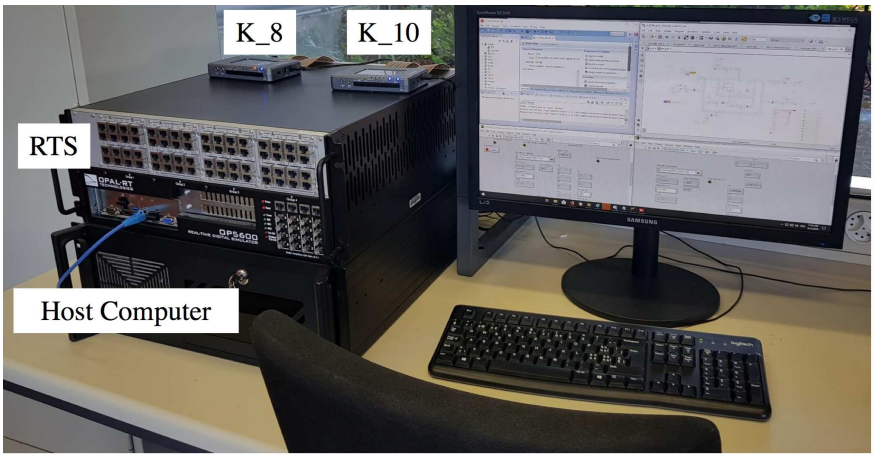

Fig. 11. HIL setup

in voltage recovery.

\section{CONCLUSION}

It has been shown how the problem of primary and secondary control design for islanded microgrids including both VSI and SG can be expressed in a $H_{2}$ framework. The proposed method directly uses the measurement data with no need for a parametric model of the system. Expressing desired performance specifications as convex constraints on sensitivity functions makes it possible to apply a convex optimization method to design the controller parameters. This results in a systematic design approach that guarantees robust stability and allows the realization of different performance objectives such as good transient performance and proportional power sharing. HIL results show that significant superior performance can be achieved as compared to classical hierarchical droop approaches. As a future work, non-linearities of the system can be considered as either frequency domain or multi-model uncertainty, which can be extracted using the measurement data in different operating points.

\section{REFERENCES}

[1] D. E. Olivares, A. Mehrizi-Sani, A. H. Etemadi, C. A. Cañizares, R. Iravani, M. Kazerani, A. H. Hajimiragha, O. Gomis-Bellmunt, M. Saeedifard, R. Palma-Behnke et al., "Trends in microgrid control," IEEE Trans. Smart Grid, vol. 5, no. 4, pp. 1905-1919, 2014.

[2] M. C. Chandorkar, D. M. Divan, and R. Adapa, "Control of parallel connected inverters in standalone ac supply systems," IEEE Transactions on Industry Applications, vol. 29, no. 1, pp. 136-143, Jan 1993.

[3] J. M. Guerrero, J. C. Vasquez, J. Matas, L. G. De Vicuña, and M. Castilla, "Hierarchical control of droop-controlled AC and DC microgridsa general approach toward standardization," IEEE Trans. Ind. Electron., vol. 58, no. 1, pp. 158-172, 2011.

[4] A. Bidram and A. Davoudi, "Hierarchical structure of microgrids control system," IEEE Trans. Smart Grid, vol. 3, no. 4, pp. 1963-1976, Dec 2012.

[5] A. Mehrizi-Sani and R. Iravani, "Potential-function based control of a microgrid in islanded and grid-connected modes," IEEE Trans. Power Syst., vol. 25, no. 4, pp. 1883-1891, Nov 2010.

[6] F. Guo, C. Wen, J. Mao, and Y.-D. Song, "Distributed secondary voltage and frequency restoration control of droop-controlled inverter-based microgrids," IEEE Trans. Ind. Electron., vol. 62, no. 7, pp. 4355-4364, 2015.

[7] J. W. Simpson-Porco, F. Dörfler, and F. Bullo, "Synchronization and power sharing for droop-controlled inverters in islanded microgrids," Automatica, vol. 49, no. 9, pp. 2603-2611, 2013.

[8] M. N. Marwali, J. Jung, and A. Keyhani, "Stability analysis of load sharing control for distributed generation systems," IEEE Trans. Energy Convers., vol. 22, no. 3, pp. 737-745, Sep. 2007.
[9] Y. A. I. Mohamed and E. F. El-Saadany, "Adaptive decentralized droop controller to preserve power sharing stability of paralleled inverters in distributed generation microgrids," IEEE Trans. Power Electron., vol. 23, no. 6, pp. 2806-2816, Nov 2008.

[10] C. Y. Chang and W. Zhang, "Distributed control of inverter-based lossy microgrids for power sharing and frequency regulation under voltage constraints," Automatica, vol. 66, pp. 85-95, 2016.

[11] J. Schiffer, R. Ortega, A. Astolfi, J. Raisch, and T. Sezi, "Conditions for stability of droop-controlled inverter-based microgrids," Automatica, vol. 50, no. 10, pp. 2457-2469, 2014.

[12] N. Ainsworth and S. Grijalva, "A structure-preserving model and sufficient condition for frequency synchronization of lossless droop inverterbased ac networks," IEEE Trans. Power Syst., vol. 28, no. 4, pp. 43104319, Nov 2013.

[13] Q.-C. Zhong and G. Weiss, "Synchronverters: Inverters that mimic synchronous generators," IEEE Trans. Ind. Electron., vol. 58, no. 4 pp. 1259-1267, 2011.

[14] N. Soni, S. Doolla, and M. C. Chandorkar, "Improvement of transient response in microgrids using virtual inertia," IEEE Trans. Power Del., vol. 28, no. 3, pp. 1830-1838, 2013.

[15] B. K. Poolla, S. Bolognani, and F. Dorfler, "Optimal placement of virtual inertia in power grids," IEEE Trans. Autom. Control, vol. 62, no. 12, pp. 6209-6220, 2017.

[16] M. Hossain, H. R. Pota, M. A. Mahmud, and M. Aldeen, "Robust control for power sharing in microgrids with low-inertia wind and PV generators," IEEE Trans. Sustain. Energy, vol. 6, no. 3, pp. 1067-1077, 2015.

[17] J. W. Simpson-Porco, F. Dorfler, and F. Bullor, "Voltage stabilization in microgrids via quadratic droop control," IEEE Trans. Autom. Control, vol. 62, no. 3, pp. 1239-1253, 2017.

[18] Q. L. Lam, A. I. Bratcu, D. Riu, and J. Mongkoltanatas, "Multi-variable H-infinity robust control applied to primary frequency regulation in microgrids with large integration of photovoltaic energy source," in Industrial Technology (ICIT), 2015 IEEE International Conference on. IEEE, 2015, pp. 2921-2928.

[19] H. Bevrani, M. R. Feizi, and S. Ataee, "Robust frequency control in an islanded microgrid: $H_{\infty}$ and $\mu$-synthesis approaches," IEEE Trans. Smart Grid, vol. 7, no. 2, pp. 706-717, 2016.

[20] M. S. Sadabadi, A. Karimi, and H. Karimi, "Fixed-order decentralized/distributed control of islanded inverter-interfaced microgrids," Control Engineering Practice, vol. 45, pp. 174-193, 2015.

[21] M. S. Sadabadi, Q. Shafiee, and A. Karimi, "Plug-and-play voltage stabilization in inverter-interfaced microgrids via a robust control strategy," IEEE Trans. Control Syst. Technol., vol. 25, no. 3, pp. 781-791, 2017.

[22] A. Karimi and C. Kammer, "A data-driven approach to robust control of multivariable systems by convex optimization," Automatica, vol. 85, pp. 227-233, 2017.

[23] S. R. Sanders, J. M. Noworolski, X. Z. Liu, and G. C. Verghese, "Generalized averaging method for power conversion circuits," IEEE Trans. Power Electron., vol. 6, no. 2, pp. 251-259, April 1991.

[24] K. De Brabandere, B. Bolsens, J. Van Den Keybus, J. Driesen, M. Prodanovic, and R. Belmans, "Small-signal stability of grids with distributed low-inertia generators taking into account line phasor dynamics," in Electricity Distribution, 2005. CIRED 2005. 18th International Conference and Exhibition on. IET, 2005, pp. 1-5.

[25] M. C. Chudasama and A. M. Kulkarni, "Dynamic phasor analysis of ssr mitigation schemes based on passive phase imbalance," IEEE Trans. Power Syst., vol. 26, no. 3, pp. 1668-1676, Aug 2011.

[26] X. Guo, Z. Lu, B. Wang, X. Sun, L. Wang, and J. M. Guerrero, "Dynamic phasors-based modeling and stability analysis of droopcontrolled inverters for microgrid applications," IEEE Trans. Smart Grid, vol. 5, no. 6, pp. 2980-2987, 2014.

[27] T. Yang, S. Bozhko, J. Le-Peuvedic, G. Asher, and C. I. Hill, "Dynamic phasor modeling of multi-generator variable frequency electrical power systems," IEEE Trans. Power Syst., vol. 31, no. 1, pp. 563-571, Jan 2016.

[28] C. Kammer and A. Karimi, "Decentralized and distributed transient control for microgrids," IEEE Trans. Control Syst. Technol., 2017.

[29] J. Löfberg, "YALMIP: A toolbox for modeling and optimization in MATLAB," in CACSD Conference, http://control.ee.ethz.ch/joloef/yalmip.php, 2004.

[30] MOSEK ApS, The MOSEK optimization toolbox for MATLAB manual. Version 7.1, 2015. [Online]. Available: http://docs.mosek.com/7.1/toolbox/index.html 